\title{
Research Experience for Undergraduates in UAV Technologies
}

\section{Dr. Subodh Bhandari, California State Polytechnic University, Pomona}

Dr. Subodh Bhandari is a professor in the Department of Aerospace Engineering at Cal Poly Pomona and the Director of its Unmanned Aerial Vehicles (UAV) Lab. His current research emphasis is on increased autonomy of unmanned aerial vehicles (UAVs), UAV-UGV collaboration, robust and intelligent control, collision and obstacle avoidance system for UAVs, coordination and control of multiple unmanned vehicles, and developing capabilities for widespread use of unmanned vehicles including precision agriculture and 3-D mapping. Dr. Bhandari leads a multi-disciplinary team of faculty and students from several departments within the Colleges of Engineering and Science at Cal Poly Pomona for research on unmanned systems. He has obtained Federal, State, and Industry support for his research on UAV's, including funding from National Science Foundation, AERO Institute/NASA Armstrong, Agriculture Research Institute, and Northrop Grumman Corporation. He is an Associate Fellow of American Institute of Aeronautics and Astronautics (AIAA) and a member of American Helicopter Society (AHS), Association for Unmanned Vehicles International (AUVSI), and American Society of Engineering Education (ASEE).

\section{Dr. Zekeriya Aliyazicioglu, California State Polytechnic University, Pomona}

Dr. Zekeriya Aliyazicioglu received his M.S. degree in 1991 and Ph.D. degree in 1995, both in Electrical Engineering from Southern Methodist University (Dallas, Texas). He is currently a Professor of the Department of Electrical and Computer Engineering at California State Polytechnic University, Pomona. His research interests include Digital Signal Processing applications, Digital Image Processing, Communication Systems, and robotics. He is an author of numerous research papers and presentations in these areas. Dr. Aliyazicioglu is a member of the Institute of Electrical and Electronic Engineers (IEEE). He is a member of Eta Kappa Nu, Tau Beta Pi and ASEE.

\section{Dr. Fang Tang, California State Polytechnic University, Pomona}

Dr. Fang (Daisy) Tang is the Chair and Professor in the Computer Science Department at California State Polytechnic University - Pomona (Cal Poly Pomona). Dr. Tang received her Ph.D. degree in computer science in 2006 from The University of Tennessee - Knoxville (UTK), performing her research on multirobot systems. She received her M.S. degree in computer science from UTK in 2003, and her B.S. degree in computer science from Sichuan University, Chengdu, China, in 2000.

\section{Dr. Amar Raheja, California State Polytechnic University, Pomona}

Dr. Amar Raheja is currently a professor of Computer Science at California State Polytechnic UniversityPomona. He received his PhD in Biomedical Engineering from University of Toledo, OH in 1999. and MS and BS from Indian Institute of Technology, India in 1992 and 1994 respectively. His research interests and publications are in the area of image processing, computer vision and machine learning. He is also a member of the IEEE and ACM. 


\title{
Research Experience for Undergraduates in UAV Technologies
}

\begin{abstract}
The Research Experience for Undergraduates (REU) in UAV Technologies Program is funded by the National Science Foundation's (NSF) Engineering Education \& Centers (EEC) Program. The main goal of this program is to increase undergraduate students' participation and interest in research on unmanned aerial vehicles (UAV) technologies. Undergraduate students from 2- and 4-year institutions are involved in a multidisciplinary research projects at the Cal Poly Pomona. The REU site supports 10 students for 10 weeks of summer research per year, with the projects focusing on research on the Dynamics and Control of UAVs, Obstacle \& Collision Avoidance System for UAVs, Machine Learning, Artificial Intelligence, Computer Vision, and Flight Test experience. Another goal is to attract students from community colleges to STEM programs at 4year institutions and encourage the participants to pursue their studies for graduate degrees.

This paper presents an overview of student activities, lessons learned so far, and the assessment of the first year of the program. The students were carefully and closely mentored by an interdisciplinary team of faculty members from various departments within the Colleges of Engineering and Science. The participating students learned to use computational tools needed to engage in multidisciplinary UAV research projects. They learned to do the scientific literature review, and had an opportunity to improve written and oral communication skills. The participants were required to present a poster, give an oral presentation of the research, and submit abstract (s) to student and/or professional conferences. In addition, the students participated in a series of research symposium and seminars designed to expose them to a range of research topics, and engage in professional development activities such as workshop on Application to Graduate Programs, Resume Building, Ethics in Engineering and Science, etc.
\end{abstract}

\section{Introduction}

UAVs have potential of replacing manned aircraft for many dull, dirty, and dangerous missions. Applications include traffic and infrastructure monitoring, surveillance of and search and rescue in disaster-hit areas, environmental gas monitoring, package delivery, aerial photography, border patrol, and precision agriculture. UAVs are cheaper than manned aircraft and pose no risk to human operators. The UAV industry is one of the fastest growing sectors of aerospace industries. However, there is a lack of professionals entering the workforce for UAV related jobs. There is also a lack of interest among undergraduates to pursue their studies for advanced degrees. The REU program at Cal Poly Pomona is designed to increase students' interest in UAV technologies by means of first-hand experience on UAV research. In addition, the students participate in a series of research symposium and seminars designed to expose them to a range of research topics, and engage in community building and professional development such as application to graduate programs, resume building, and ethics in engineering and science. They are encouraged to present their research at conferences and during symposiums. 
This paper presents a brief overview of the research and professional development activities that the students are involved in. The paper also presents the lessons learned so far, as well as the evaluation of the first year of the program.

The following paragraphs discuss the research environment for the participants, some sample projects that the participants are involved in, professional development opportunities for the participants, and outcomes and evaluation of outcomes followed by the conclusion and future work.

\section{Research Environment}

In the beginning of the summer program, the students are introduced to the research environment and research projects that they will be involved in by the research supervisor. The students are usually teamed up in a group of 2 or more. The students are asked to do literature review, and come up with a solution for the problems that need further development and maturation. The students are highly encouraged to present their work during student and professional conferences. All this provides an intensive research environment where the students learn, by means of handson learning, the state-of-the-art in UAV technologies. The students also attend weekly research seminars held at Cal Poly Pomona, and meet with their research supervisors every week.

Cal Poly Pomona is currently engaged in several UAV research projects. The ongoing research projects include development and validation of flight dynamics models of UAVs, ${ }^{1}$ modeling and simulation,$^{2,3}$ development of obstacle and collision avoidance capabilities ${ }^{4,5,6}$, vegetation growth analysis using UAVs, ${ }^{7}$ and search and rescue using UAVs. ${ }^{8}$ An excellent collection of UAVs and associated equipment at the university facilitates these projects. The UAV Lab at Cal Poly Pomona has more than 25 fixed- and rotary-wing UAVs of various sizes and payload capacities. Some of the UAVs are shown in Figure 1. Also available for the research projects are autopilots, inertial measurement units (IMUs), Differential global Positioning System (DGPS), stabilized camera gimbal, multispectral and hyperspectral sensors, Light Detection and Ranging (LIDAR) sensors, and infrared cameras.

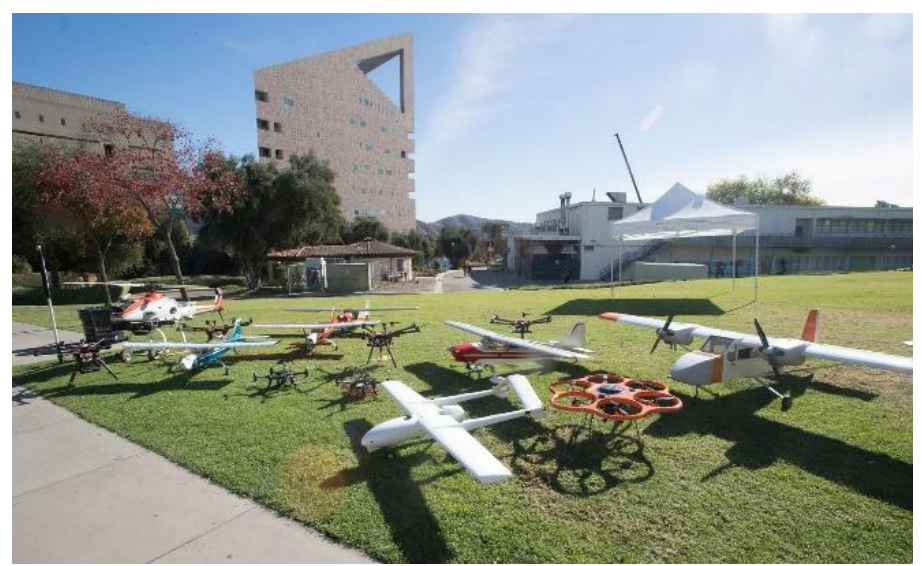

Figure 1. UAV fleet at Cal Poly Pomona.

The projects usually address or try to address the problems that the UAV operations is currently facing such as integration of the UAVs into the National Airspace System, Collision and Obstacle 
Avoidance, Navigation in GPS-Denied Indoor Environments, Artificial Intelligence, and Modeling and Simulation. During the Program period, the students work on sensor and processor selection and integration, algorithm development, flight testing, data collection, and data analysis.

\section{Development of Technical Expertise}

The goal of the program is to involve the REU participants in the UAV related cutting-edge research projects. The projects are being designed to increase students' interest in UAV technologies, engineering, and computer science, and develop their research skills. The following paragraphs describe the projects that the 2017 summer REU participant were involved in. The students were provided with an opportunity to gain knowledge on the application of computer science and engineering to UAV technologies and were able to acquire some of the skills necessary to conduct meaningful research in UAV technologies, understand research process, and learn laboratory techniques. Most of the projects described below are highly interdisciplinary. Each REU student had a primary mentor. However, the students were co-supervised by other mentors depending on the nature of the projects.

\section{A. Autonomous Collision Avoidance of UAVs Utilizing ADS-B Transponders}

For the UAVs to be successfully integrated into the U.S. national airspace, the ability to detect and avoid both manned and unmanned aircraft is a necessity. This project involved the students in developing a method for collision avoidance using Automatic Dependent Surveillance - Broadcast (ADS-B) transponder. ${ }^{9}$ These devices broadcast and receive global position of all similarly equipped aircraft in a 100-nautical mile vicinity. A Sig Kadet Senior and a Hangar 9 Valiant fixed wing aircraft are used as UAV platforms to test autonomous collision avoidance. The UAVs are equipped with Pixhawk autopilot and an Intel NUC as the primary components of the avionics system. The developed collision avoidance algorithm uses kinematic and circular motion equations to predict the future positions of both aircraft and employ an avoidance maneuver at a constant altitude. The uniquely developed algorithm was compiled in a Linux environment, and used MAVLink protocols to send off-board commands to the autopilot. The system was demonstrated through simple ground tests, and subsequently moving on to full-system flight tests. Figure below on the left shows a group of students engaged in flight tests to demonstrate collision avoidance. The figure on the right shows the flight path of the UAV after it avoided a simulated obstacle. The students involved in this project presented their work at the American Institute of Aeronautic and Astronautics' (AIAA) SciTech Forum. ${ }^{10}$
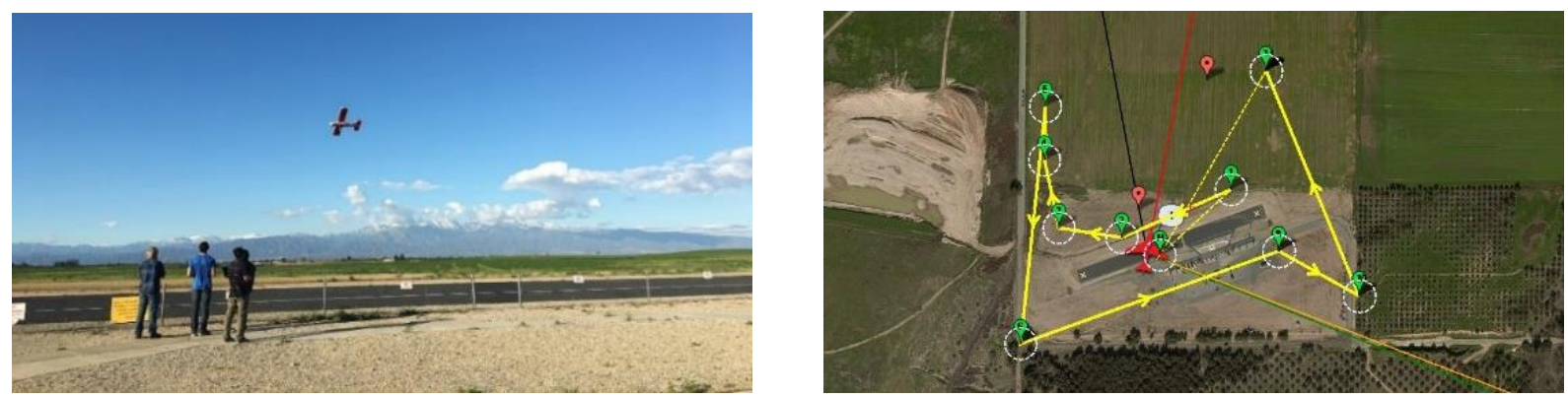

Figure 2. Flight testing (left) and UAV path after a simulated obstacle was avoided by the UAV (right). 


\section{B. Search and Rescue using Multiple UAVs}

UAVs can be cost-effectively and efficiently used for indoor search and rescue missions. These environments pose dangerous and risky scenarios for rescue personnel. UAVs can locate and assist victims that are in need during the event of natural disaster with increased safety and low response time, without posing any danger to the rescuers. However, the lack of GPS signal in the indoor environments poses many difficulties for the use and navigation of these systems. A search UAV, a quadcopter equipped with a Pixhawk autopilot, uses a front-facing camera for the target detection, and an ultrasonic sensors for obstacle detection. Using computer vision and machine learning, the search quadcopter can navigate through the indoor environments and identify survivors of disaster, and then relay this information to the rescue UAV, also a quadcopter, via a ground control station (GCS). The rescue quadcopter is designed to navigate to the location of the victim and releases the payload. The use of multiple UAVs allows for smaller, lighter, and more agile solutions to perform the tasks more efficiently. Figure 3 on the left shows the search UAV, and figure on the right shows the rescue UAV.
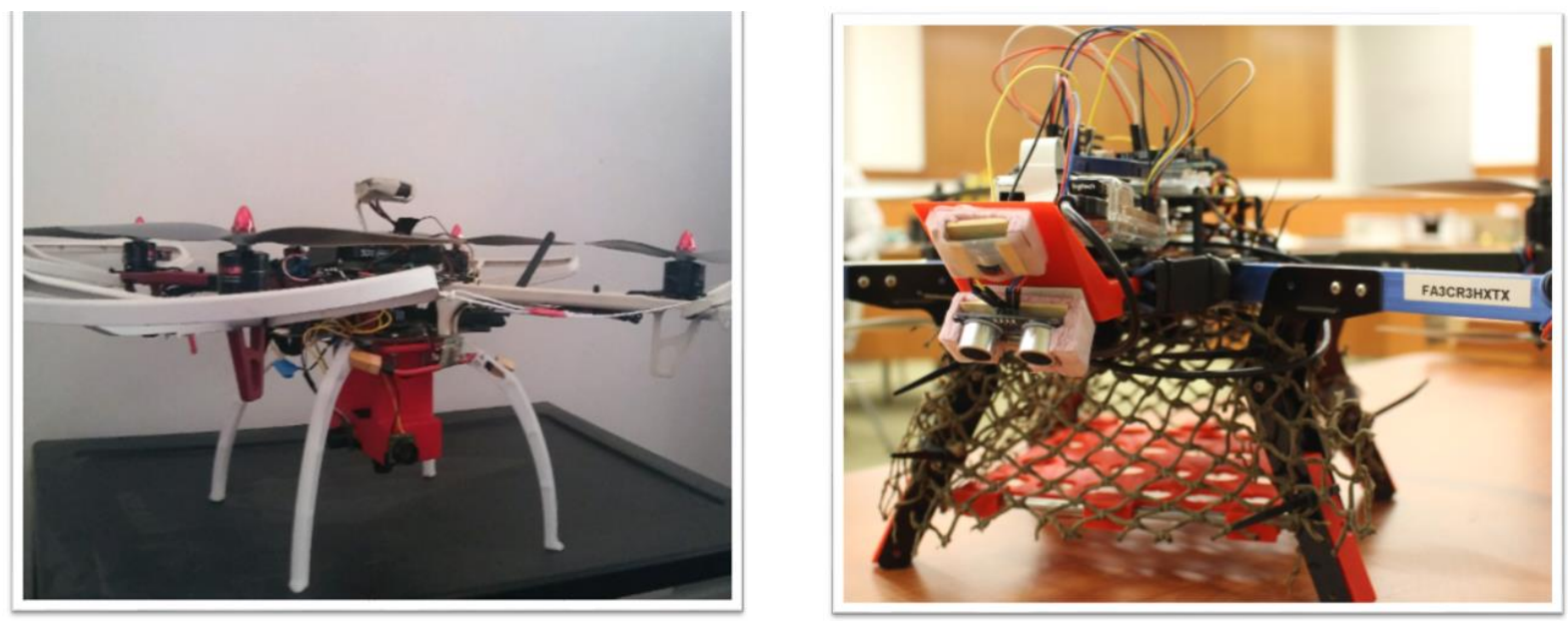

Figure 3. Search quadcopter (left) and rescue quadcopter (right).

\section{3-D Mapping using Unmanned Aerial Vehicles}

This project uses a DJI S1000 octocopter equipped with an autopilot for autonomous waypoints operation, a VPL-16 LIDAR for the surface data required for 3-D mapping, a Global Navigation Satellite System (GNSS) aided inertial navigation system (INS) for tracking the location and orientation of the LIDAR and the UAV, and an Intel NUC processor board, which processes the LIDAR data. The UAV was structurally modified to attach a 3-D printed LIDAR mount at a $40^{\circ}$ inclination for the optimum results while keeping the center of gravity at an acceptable position. The raw LIDAR data is exported to LAStools for 3-D visualization. ${ }^{11}$ In order to improve the quality of the 3-D maps, the LIDAR data is combined with the GNSS aided INS data. Figure 4 on the left shows the UAV used in this project, and the figure on the right show the 3-D model of citrus trees generated using the LIDAR data. The students involved in this project presented their work at the AIAA SciTech Forum. ${ }^{11}$ 

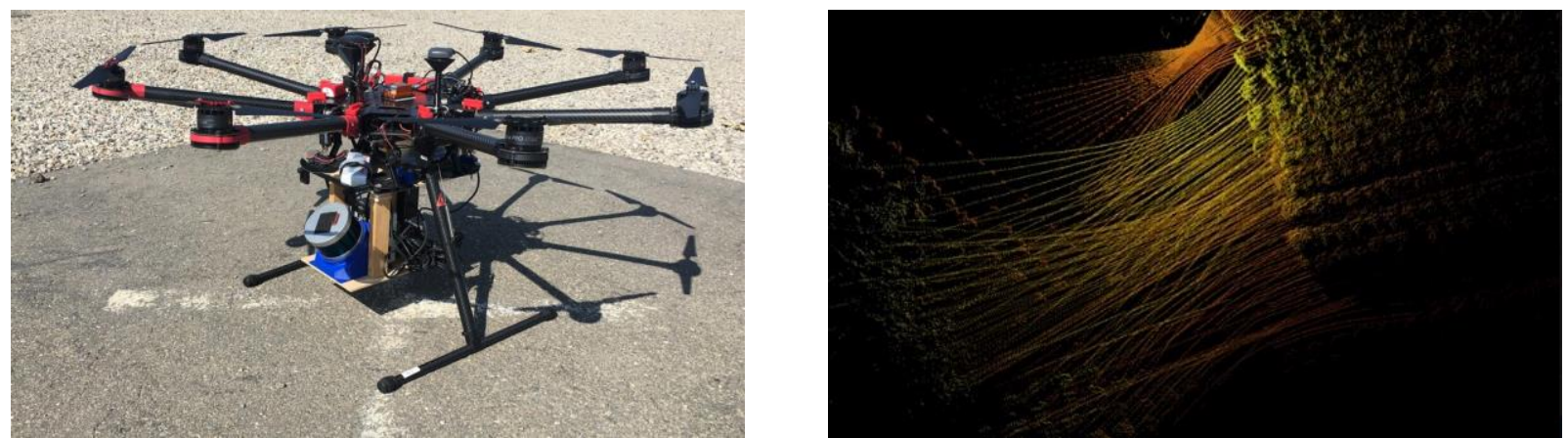

Figure 4. DJI S1000 equipped with LIDAR (left) and 3-D model of citrus trees (right).

\section{IV.Professional Development of Participants}

For the professional development of the participants, several activities were planned. This REU program seeks to train undergraduates who are not only skilled in their technical areas, but also who can effectively present their research to both technical and non-technical audiences. Several workshops were conducted during the 10- week period that included Ethics in Engineering and Science, Graduate School Application Process and Financial Support, Resume Building, Improving Oral and Written Presentation Skills, and Industry Careers.

We sought to enhance the students' communication skills by engaging them in outreach activities to the general public and K-12 students. The REU students were asked to make a presentation on the UAV technologies to K-12 students. Figure 5 shows some of the REU participants giving a presentation to some local middle school students.

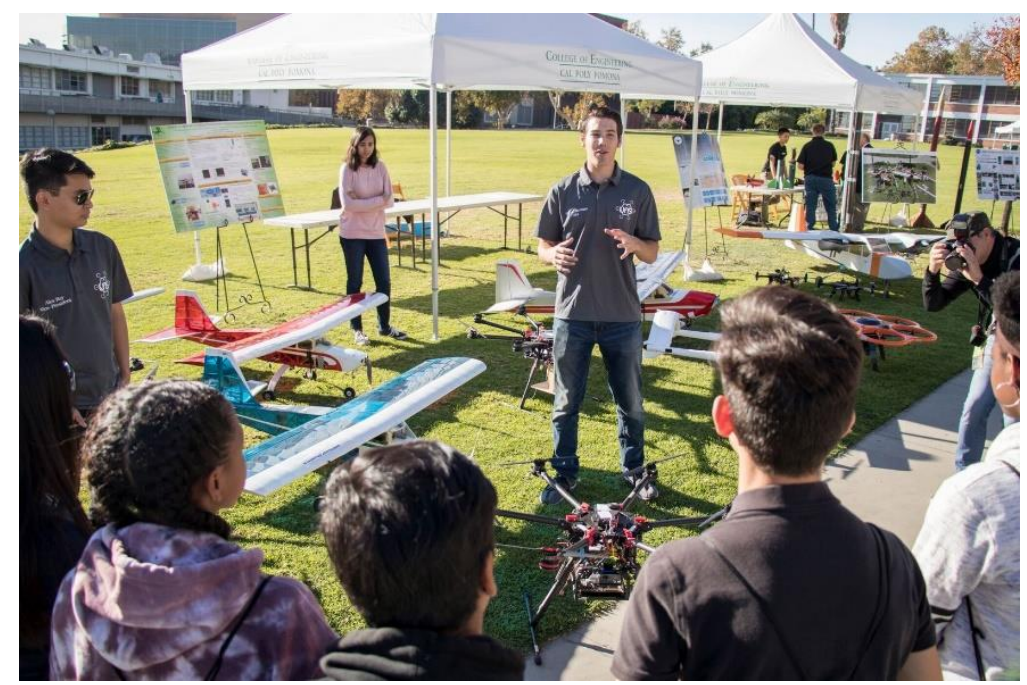

Figure 5. REU participants engaged in outreach activities.

To further enhance the development of the REU participants into outstanding scholars, all participants were strongly encouraged to present their work at the student and professional conferences. All of the participants presented their work during the Creative Activities and Research Symposium (CARS) organized at Cal Poly Pomona at the end of the Program. They also presented their work at the Southern California Conference for Undergraduate Research 
(SCCUR). One of the participants was selected for and presented his work at the REU Symposium organized by the Council on Undergraduate Research. Some of the participants presented their work at the AIAA SciTech 2018 Forum, which is a professional conference organized by AIAA. ${ }^{10,11}$ A paper submitted by two other participants to the International Conference on Unmanned Aerial Systems (ICUAS) has been accepted for presentation at and publication in the conference proceedings. ${ }^{12}$ Rest of the participants have submitted papers or are preparing papers for submission to other professional conferences for presentation at and publication in the conference proceedings.

\section{Outcomes and Evaluation of Outcomes}

The outcomes of the first year of the Program was assessed through the extent to which the following Program goals were achieved: 1) Increased interest among the participants in UAV Technologies; 2) Knowledge of flight dynamics and control of UAVs; 3) Recognition of the need for, and an ability to engage in life-long learning; 4) Improvement of written and oral communication skills; 5) Opportunity to learn other disciplines; and 6) Interest among students to pursue graduate degrees.

The evaluation of the Program was done by an external evaluator not involved with the REU Program. The evaluator conducted pre- and post-participation surveys from the Participants as well as collected the faculty mentor evaluations of the participants.

\section{A. Evaluation of Student Learning}

Pre-program surveys assessed the students' interest in the UAV technologies, knowledge of the UAV technologies (a content knowledge measure), and the students' attitudes toward and commitment to ongoing learning. During the last week of the summer Program, the evaluator had a second meeting with the participants. The post-test reassessed areas covered in the initial evaluation as well as included free-response items for the students to provide qualitative feedback on their experiences in the REU Program.

In general, the participants expressed overall satisfaction with the program and gratitude for their experiences. Some of the comments include: 1) "The UAV program impacted me in a very positive manner," 2) "My experience here was good. I appreciate that we were taken care of with the housing \& food. Our advisor was very friendly as well and made us feel comfortable," 3) "I would highly recommend this program to everyone interested in UAVs," and 4) "The best part was working with my team to complete our goal."

One aspect of the program that participants particularly emphasized as contributing to their enjoyment and learning was the interpersonal relationships they developed with the fellow participants. They mentioned that the best aspect was the team-building experience, living together, working together, and facing challenges together.

The participants also described a feeling that they had learned a great deal during the Program. Several participants mentioned that they felt that they learned a lot that was directly related to the 
UAV technologies and computer science. They liked the opportunity to work on an actual team project and to learn more about testing and analyzing data in a multidisciplinary environment. Some of the participants mentioned that they knew very little about UAVs and that their technical knowledge helped them understand what they needed to learn to solve these complex problems. It gave confidence to the participants to take a very complex problem and still succeed at the end.

The participants also described that they learned transferable skills that they would carry forward to new projects. They also appreciated the opportunity to learning new skills, team building, and other practical skills that they can now apply to other projects. Many participants did indicate that the program led them to feel more certain or clear about their careers plans, and applying to graduate programs.

Figure 6 shows the student responses to pre- and post-participation survey questionnaire. It is seen that the average participant responses improved significantly post-participation compared to the pre-participation responses.

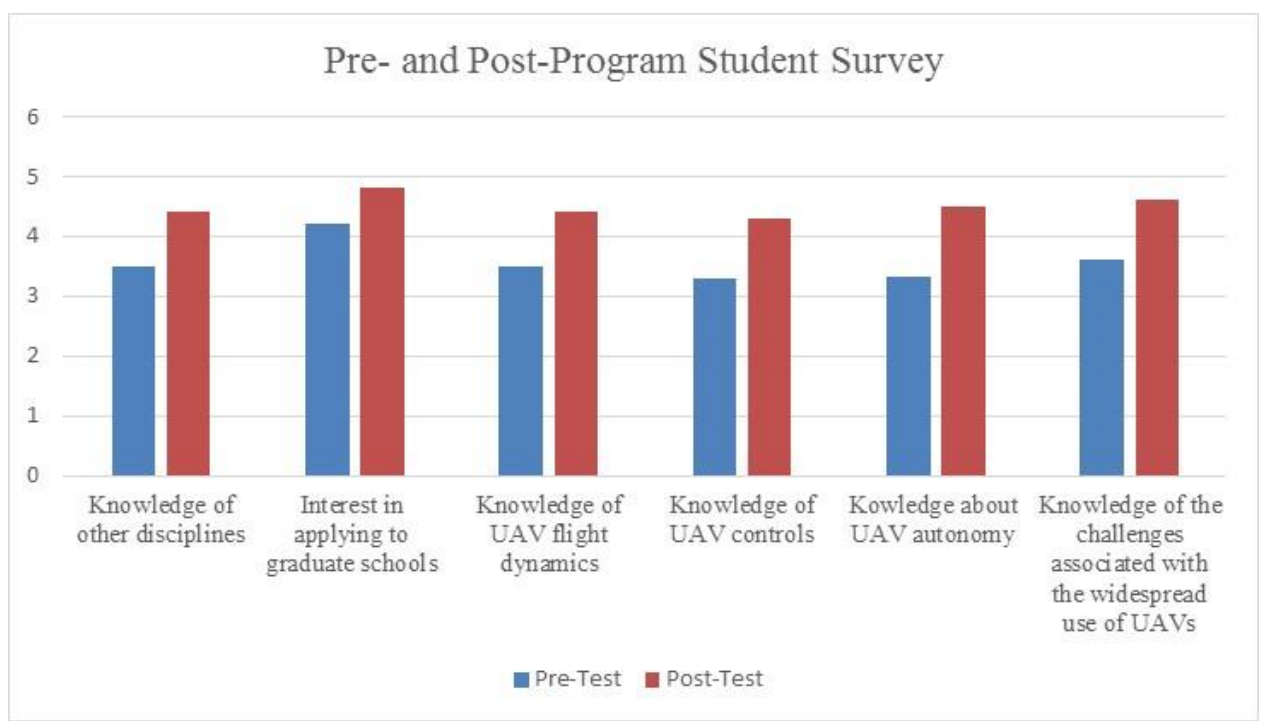

Figure 6. Student responses to pre- and post-participation survey questions.

\section{B. Participants' Recommendation for Improvements}

While the participants' comments were generally very positive, the one recommendation for improvement was the need for more emphasis on and communication of the Program structure before and in the first week of the Program. The participants also expressed that they would benefit from industry exposure. Though some field trips were planned, they had to be cancelled due to last minute changes.

\section{Mentor Feedback}

The evaluator also met with the faculty mentors during the last week of the Program. The faculty mentors provided feedback regarding the students' capacity for self-directed learning and original 
investigation as well as the participants' skills for communicating scientific and engineering concepts. The mentors also provided feedback about their involvement in the Program. Figure 7 shows the mentor evaluations of the participants at the start and end of the Program.

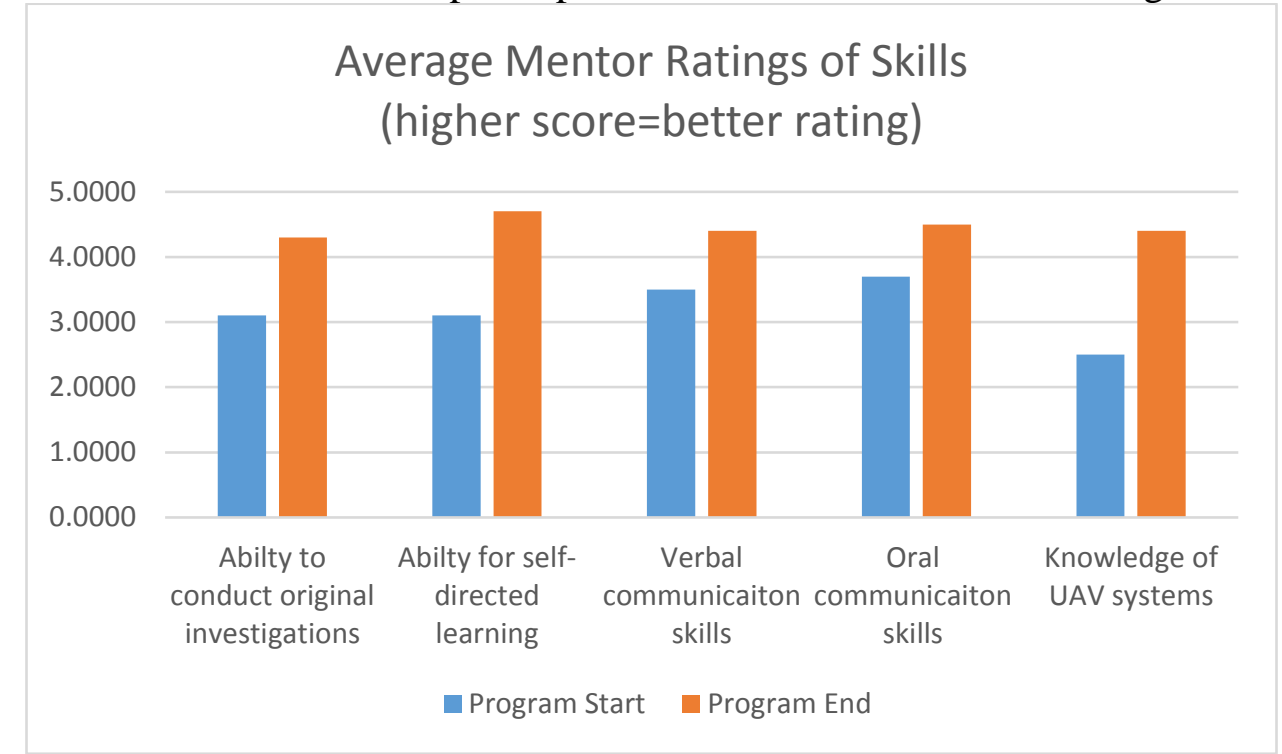

Figure 7. Mentor evaluation of the REU participants.

Comparing the mean ratings of the student skills at the start and end of the program (collected retrospectively at the end of the program), mentor evaluations indicated statistically significant changes in the average skills ratings for the participants in the course of the program.

Mentors echoed the student comments that they enjoyed the Program and particularly emphasized that they liked directly working with the students on the research projects. For example, one mentor wrote that, "the best aspect was advising the students and seeing them being excited about learning the science and engineering." All the mentors reported that they collaborated with the other mentors on the project.

\section{Conclusion and Future Work}

Overall, the UAV REU Program is on target to meet the goals of the Program. The Program participants were diverse with regard to race and ethnicity. Forty percent of the participants were Hispanic or Latino. The program also included women, although they comprised only $20 \%$ of the cohort.

Faculty mentors' evaluations showed significant gains for the participants in the development of students' ability to engage in life-long learning by increasing the capacity for self-directed learning and original investigation. The participants particularly highlighted the collaborative nature of the work as a favorite part and major strength of the Program. There were significant improvements in skills for communicating scientific and engineering concepts via written and oral methods. Participants also showed increased interest in pursuing graduate degrees. 
In the future years, we will continue to foster the recruitment of an ethnically diverse groups of participants, and consider targeted efforts to recruit more women into the program. We will plan for field trips to the local research labs and industry in advance so that the participants get some industry exposure.

\section{Acknowledgement}

The project is funded by the NSF's EEC Program. We would like to thank the external evaluator for her help with conducting the evaluation of the Program, and several others who were directly and indirectly involved with the project. We would also like to thank Northrop Grumman Corporation, The Boeing Company, Lockheed Martin, and NASA Armstrong for their continued support of the Cal Poly Pomona's UAV Lab.

\section{References}

1. Anderson, N., Hagenauer, B., Erickson, R., and Bhandari, S., "Flight Testing of a UAV Airplane for Autonomous Operation using Piccolo II Autopilot," Proceedings of AIAA Atmospheric Flight Mechanics Conference, Honolulu, HI, 18-21 Aug. 2008.

2. Gomez, E., Garcia, D., Piana, M., Ritterbush, M., and Bhandari, S., "Simulation Environment for Testing UAS Collision Avoidance System," Proceedings of Infotech@Aerospace Conference, San Diego, CA, 4-8 Jan. 2016.

3. Navarro, P., Cho, S., Rashid, A., Ruiz, A., and Bhandari, S., "Flight Testing, Data Collection, and System Identification of a Multicopter UAV," Proceedings of Infotech@Aerospace Conference, Grapevine, TX, 9-13 Jan. 2017.

4. Richards, B. Gan, M., Bhandari, S., et al., "Collision Avoidance System for UAVs using Computer Vision," Proceedings of AIAA Infotech@Aerospace, Kissimmee, FL, January 5-9, 2015.

5. Wagster, J., Bhandari, S., et al., "Obstacle Avoidance System for a Quadrotor UAV," Proceedings of AIAA Infotech@Aerospace, Garden Grove, CA, June 19-21, 2012.

6. Bhandari, S., Rose, M., et al., "Tracking of Mobile Targets using UAVs," Proceedings of AIAA Guidance, Navigation, and Control Conference Invited Session, Minneapolis, MN, Aug. 13-17, 2012.

7. Ding, K., Raheja, A., and Bhandari, S. "Application of Machine Learning for the Evaluation of Turfgrass Plots using Aerial Images," To Appear in the Proceedings of SPIE Autonomous Air and Ground Sensing Systems for Agricultural Optimization and Phenotyping Conference, Baltimore, MD, 17-19 Apr. 2016.

8. Bhandari, S., Bettadapura, A., Dadian, O., et al., "Search and Rescue using Unmanned Aerial Vehicles," Proceedings of AIAA Infotech@Aerospace, Kissimmee, FL, 5-9 Jan. 2015.

9. Schaeffer, R., "A Standards-Based Approach to Sense-and-Avoid Technology," AIAA 3rd Unmanned Unlimited Technical Conference, Workshop and Exhibit, Sept. 2004.

10. Sherman, T., Tellez, J., Haideri, H., Lopez, J., Bhandari, S., et al., "Cooperative Search and Rescue using Autonomous Unmanned Aerial Vehicles," Proceedings of Infotech@Aerospace Conference, AIAA SciTech Forum, Kissimmee, FL, 8-12 Jan. 2018.

11. Carreon-Limones, C., Asher, M., Ruiz, V., Lauf, R., and Bhandari, S., "Increasing the Accuracy of 3D Maps Created using a UAV-Based LIDAR," Proceedings of Infotech@Aerospace Conference, AIAA SciTech Forum, Kissimmee, FL, 8-12 Jan. 2018.

12. Perez, E., Winger, A., Bhandari, S., et al., "Autonomous Collision Avoidance System for a Multicopter using Stereoscopic Vision," Accepted for Presentation at and Publication in the Proceedings of International Conference on Unmanned Aerial Systems, Dallas, TX, 12-15 June 2018. 